\title{
Projeto e Análise de Controladores Robustos aplicados a Moagem na Mineração*
}

\author{
Felipe F. de Castro*,**, Thiago A. M. Euzébio*, \\ Márcio F. Braga ${ }^{*, * *}$ \\ * Instituto Tecnológico Vale - ITV, Ouro Preto, MG, Brasil \\ ** Departamento de Engenharia Elétrica, Instituto de Ciências Exatas \\ e Aplicadas, Universidade Federal de Ouro Preto - UFOP, \\ 35931-008, João Monlevade, MG, Brasil. \\ E-mails: felipefuscaldi@gmail.com, thiago.euzebio@itv.org, \\ mfbraga@ufop.edu.br
}

\begin{abstract}
The PI and PID controllers are widely used in industry. It is proposed to compare classical PI controllers with robust PI controllers in a mining grinding process. Strategies are tested using process simulation software and control for evaluation. The comparison verified how fast and adherent the control loop outputs are if compared to the reference, even though the process has uncertainties and disturbances. We conclude this study by presenting the results of the simulations and an analysis of these results.

Resumo: Controladores PI e PID são amplamente utilizados na indústria. Neste artigo, propõese a comparação de controladores PI clássicos com controladores PI robustos em um processo de moagem na mineração. As estratégias são testadas por meio de um software de simulação de processo e controle para avaliação. A comparação verificou quão rápidas e aderentes são as saídas das malhas de controle se comparadas à referência, ainda que o processo tenha incertezas e distúrbios. Conclui-se este estudo apresentando os resultados das simulações e uma análise dos resultados, assim como apresentam-se propostas de trabalhos futuros.
\end{abstract}

Keywords: Process Control; Grinding; PID; Robust; Ball Mill; LMI

Palavras-chaves: Controle de Processos; Moagem; PID; Robusto; Moinho de Bolas; LMI;

\section{INTRODUÇÃO}

No processo de beneficiamento do minério de ferro, a moagem é uma das etapas mais críticas de produção e de maior consumo energético conforme Castro e Braga (2018). Tais fatos abrem caminhos para pesquisas em otimização, eficiência e controle do processo, com o objetivo de alcançar maior economia e qualidade do produto a ser gerado nesta etapa (Castro e Braga, 2018).

O controle ineficiente desse circuito contribui para a moagem em excesso do material, o que também provoca o aumento desnecessário do consumo energético. Por exemplo, na Samarco, as etapas de moagem representam aproximadamente $80 \%$ do consumo de energia, sendo que desse total, a remoagem do concentrado é responsável por $60 \%$. O material mais fino do que o especificado gerado na moagem dificulta a recuperação das partículas no processo de concentração. Esse material também prejudica a operação de filtragem do processo de pelotização devido a entupimentos (Reis, 2018).

A moagem é um processo complexo com pelo menos três aspectos significativos. O primeiro refere-se aos equipamentos do processo, como moinho de bolas, ciclone, polpa

\footnotetext{
* Os autores agradecem às agências CAPES, CNPq, à Universidade Federal de Ouro Preto (Número do Processo 23109.003268/2017-47) e ao Instituto Tecnológico Vale (ITV) pelo apoio financeiro.
}

de lama, válvulas, dentre outros, que são dispositivos dinâmicos e resultam em maior complexidade no controle. $\mathrm{O}$ segundo relaciona-se aos muitos fatores que são aleatórios e de grande incerteza, o que tornam o processo não linear (Hongwei et al., 2010). O terceiro está ligado aos desafios ao controlar um processo de moagem que são o forte acoplamento entre variáveis, grandes atrasos no tempo, perturbações incontroláveis, variação de parâmetros ao longo do tempo, as não linearidades do processo e inadequações de instrumentação (le Roux et al., 2014).

O controle robusto aplicado ao processo de moagem foi estudado por Su et al. (2009), por Jenkins et al. (1996) e por Galán et al. (2002). Su et al. (2009) propõem dois controladores robustos para uma planta de moagem de carvão. O primeiro é baseado em RFN (do inglês, Reverse Frame Normalized) e o segundo baseado na norma $\mathscr{H}_{\infty}$. Jenkins et al. (1996) apresenta que o controle da posição, velocidade e força são críticos para obter um produto de alta qualidade na moagem. São propostas duas malhas de controle separadas sendo uma malha de velocidade de alimentação padrão e uma malha de força. Em Galán et al. (2002), os autores estudam o controle robusto em moinhos semiautógenos e apresentam um projeto de controle robusto para uma usina de moagem semiautógena (SAG). São propostos controladores robustos que realizam um 
controle rígido sobre o consumo de energia do moinho SAG a partir do ajuste da taxa de alimentação do moinho.

Dentro dessa proposta, este artigo apresenta uma comparação para o emprego de controladores PI (Proporcional e Integral) clássicos e robustos, utilizando o modelo do processo de moagem do Concentrador 3 da mineradora Samarco. Os controladores robustos utilizam técnicas baseadas em desigualdades matriciais lineares (LMI, do inglês Linear Matrix Inequality). O objetivo é mostrar que, em processo sujeitos a varições e incertezas, controladores robustos garantem a estabilidade do processo e são capazes de mitigar os efeitos da variação dos parâmetros que ocorrem ao longo do tempo. Destaca-se ainda que, na prática, a proposta não envolve o aumento de custos, pois sua aplicação implica em apenas alterar os ganhos dos controladores PI atualmente presentes no processo.

\section{DESCRIÇÃO DO PROCESSO DE MOAGEM}

\subsection{Descrição do Processo de Moagem do Concentrador 3 da Samarco}

Reis et al. (2018) descreve o processo de moagem, o qual é utilizado neste estudo como planta modelo. Também chamado de moagem secundária, ou remoagem, o processo é alimentado pelo concentrado do circuito de flotação com uma vazão nominal de $1455 \mathrm{t} / \mathrm{h}$ por linha.

Os dois moinhos trabalham em paralelo, em circuito inverso com ciclones de $0,25 \mathrm{~m}(10 ")$. A pré-classificação é feita em circuito aberto por meio de uma bateria com 18 ciclones, onde o número de ciclones em operação depende da pressão na alimentação. Na Figura 1, é mostrado um fluxograma do processo de remoagem da Samarco do qual destacam-se o moinho de bolas, os hidrociclones 01, 02 e 03 e as bombas de polpa 01 e 02 .

Baseado nos estudos de Chen et al. (2009) para moagem, foi proposto por Reis (2018) quatro malhas para o controle de vazão utilizando controladores PI que se adaptam ao estudo da remoagem. A primeira malha é identificada como SIC-004 e controla a vazão da bomba 01; a segunda malha, FIC-013, controla a vazão de água no moinho de bolas; a terceira, LIC-001, controla o nível da caixa de descarga e, por fim, a malha SIC-001 que controla a vazão da bomba 02. Desgastes devido ao uso do equipamento, à falta ou à manutenção precária e até mesmo uma modelagem ruim do processo real podem justificar a aplicação de um controlador robusto. A seguir apresentam-se os três principais equipamentos que são citados como mais importantes e que possuem variações nos parâmetros (Chen et al., 2009).

Moinho de Bolas. O moinho de bolas é largamente utilizado na mineração de ferro. Na moagem via úmida, que é uma das características do circuito de moagem estudado, o material é misturado com água suficiente para formar uma polpa na entrada do moinho. Das variáveis de processo importantes desse equipamento, podem-se destacar a potência do motor e o percentual de sólidos da polpa que alimenta o moinho. O percentual de sólidos é controlado com a adição de água na alimentação, que depende da granulometria do material da alimentação e do tipo do moinho. O controle da potência do moinho é comumente realizado a partir da adição de bolas para

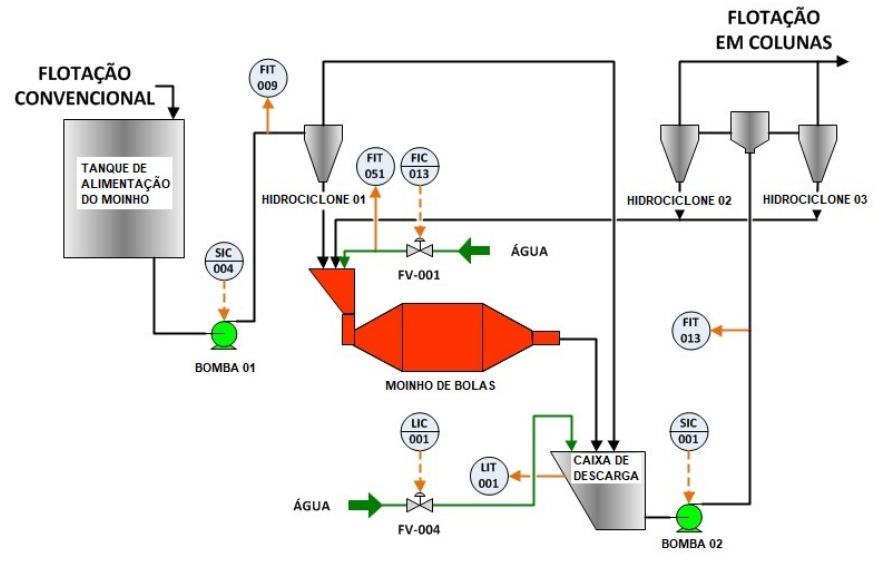

Figura 1. Fluxograma do processo de remoagem do concentrador 3 da Samarco (Adaptado de Reis (2018)).

regular a potência ideal de operação sempre que a potência ficar abaixo de um limite preestabelecido (Reis, 2018).

Hidrociclones. Segundo Reis (2018), as propriedades geométricas do ciclone, as propriedades físicas dos materiais que compõem a polpa e as condições operacionais são fatores que influenciam no desempenho do equipamento. As variáveis operacionais dos hidrociclones que podem ser controladas para seu melhor desempenho na classificação são o percentual de sólidos e a pressão da alimentação. O percentual de sólidos influencia significativamente o diâmetro de corte e a pressão no hidrociclone. Essa, por sua vez, pode ser controlada a partir da abertura ou fechamento de hidrociclones.

Os modelos matemáticos propostos na literatura para aplicações em hidrociclones são apenas aproximações da realidade física e, por isso, possuem limitações, o que quer dizer que erros de dados medidos são incluídos no modelo e, consequentemente, na previsão gerada (Silva, 2014).

Bombas de Polpa. Os equipamentos auxiliares presentes no processo de moagem são as bombas centrífugas que podem aparecer na alimentação e descarga do moinho ou acopladas em uma caixa de polpa (Reis, 2018).

As principais variáveis para o controle desses equipamentos são a vazão no recalque e a velocidade. Nas plantas de beneficiamento mais modernas, essas bombas possuem inversores de frequência que possibilitam a variação da velocidade. Assim, o sistema de controle das bombas centrífugas tem o objetivo de manter uma vazão de polpa estável por meio da variação da velocidade (Reis, 2018).

Variações na Moagem. O moinho de bolas é o principal equipamento do processo e as variações que ocorrem nele afetam fortemente a moagem. Assim Rockwell Automation (2016) cita as variações no processo que podem ocorrer devido a um comportamento oscilatório e desvio do set-point; controle ineficiente da qualidade do produto (granulometria); desgaste desnecessário do moinho (aço com aço) e o moinho não operando com máxima eficiência energética. Cita ainda que os objetivos do controle na moagem são o controle da granulometria do produto próximo a $P_{80}$ (abertura em malha quadrada que deixa passar $80 \%$ do produto); manter a pressão de entrada no hidrociclone 
abaixo dos limites toleráveis; manter nível do reservatório dentro da faixa de operação e manter a corrente elétrica da bomba de circulação abaixo dos limites. Reis (2018) ainda cita três situações comuns na operação da planta. A primeira que está relacionada a mudança do tipo de material na alimentação do moinho devido às diferentes lavras do minério e outras duas que se referem ao percentual de minério na polpa de alimentação. Tais objetivos de controle na moagem e as variações que podem ocorrer durante o processo permitem que o uso do controle robusto tenha sucesso quando aplicado a esse tipo de processo.

\subsection{Simulação do Processo de Remoagem}

Légaré et al. (2016) afirma que a simulação do processo é uma ferramenta atraente para se projetar, avaliar e précomissionar estratégias de controle avançado. Afirma também que a programação feita por blocos permite simular várias situações com mais facilidade do que em plantas reais e ainda são flexíveis para modificações futuras ou atualizações de modelo.

Simuladores dinâmicos são mais apropriados para avaliar o impacto de variabilidade de entrada e desempenho de controle de processo (Légaré et al., 2016). O software industrial de simulação de processos da Andritz Automation IDEAS $^{\text {TM }}$ é utilizado por Légaré et al. (2016) na simulação de um circuito dinâmico de moagem e também por Sbárbaro e del Villar (2010). Guerrero et al. (2016) utiliza o IDEASTM como software de simulação de processos minerais. Publicações que também utilizam o $\operatorname{IDEAS}^{\mathrm{TM}} \mathrm{em}$ processos de mineração são Magalhães e Euzébio (2018) e Lopes Jr. et al. (2018). Outro software empregado neste estudo e amplamente utilizado como simulador e controlador de processo é o MATLAB/Simulink ${ }^{\circledR}$ do qual Guerrero et al. (2016) utiliza em um processo de controle de um moinho SAG e realiza a interface entre $\operatorname{IDEAS}^{\mathrm{TM}}$ e MATLAB/Simulink ${ }^{\circledR}$ via OPC (do inglês, OLE for Process Control).

\section{PROJETO DE CONTROLADORES PI EMPREGANDO LMI}

O problema a ser resolvido neste trabalho consiste em determinar ganhos $K_{p}$ e $K_{i}$ para um controlador PI em um circuito de retroalimentação negativa que controle as malhas do circuito de moagem. São apresentadas cinco metodologias para o cálculo dos ganhos, dentre as quais três metodologias são clássicas e as outras duas são de controladores robustos.

\subsection{Descrição e Modelagem do Sistema}

A identificação das malhas foi realizada com erro máximo de $8 \%$ para o pior caso e resultou em modelos de primeira ordem que apresentam pequenos atrasos de transporte na ordem de $2 \mathrm{~s}$, descritos genericamente por

$$
G(s)=\frac{K}{a s+1} e^{-\tau s} .
$$

Os modelos das malhas estão listados na Tabela 1. Para a identificação dos modelos foi utilizando um sinal PRBS (do inglês, Pseudo Random Binary Signal) aplicado ao modelo validado do IDEAS ${ }^{\mathrm{TM}}$ e a identificação do sistema via System Identification do MATLAB ${ }^{\circledR}$. Confirmou-se o descrito por Reis (2018) que parte dos problemas de controle na moagem estão ligados a sistemas com atrasos de transporte. Os modelos apresentados na Tabela 1 foram utilizados para o cálculo dos ganhos dos controladores.

Tabela 1. Modelos identificados para as malhas estudadas.

\begin{tabular}{cc}
\hline \hline Malha & Função de Transferência \\
\hline SIC-004 & $\frac{16,7608}{2,7174 s+1} e^{-2 s}$ \\
\hline FIC-013 & $\frac{2,1045}{1,7895 s+1} e^{-1,8 s}$ \\
LIC-001 & $\frac{11,6279}{1,0668 s+1} e^{-2,5 s}$ \\
\hline SIC-001 & $\frac{36,5620}{2,4522 s+1} e^{-2 s}$ \\
\hline \hline
\end{tabular}

\subsection{Metodologias Clássicas}

Foram testadas algumas metodologias clássicas de controle de processos como o Método da Integral do Erro Absoluto multiplicado pelo Tempo (ITAE), Método Cohen \& Coon, Método CHR proposto em Chien et al. (1952), Método do Modelo Interno aplicado para o controle robusto (IMCR) descrito em Seborg et al. (2010) e também a sintonia SIMC proposta por Skogestad (2003). Após realizadas as simulações, foram escolhidos os três métodos dentre os citados que obtiveram os melhores desempenhos, considerando menor sobressinal e menor tempo de assentamento. As metodologias escolhidas foram a SIMC, ITAE e IMC-R cujos valores de sintonia estão apresentados na Tabela 2.

Tabela 2. Ganho dos Controladores Clássicos.

\begin{tabular}{c|cc|cc|cc}
\hline \hline \multirow{2}{*}{ Malha } & \multicolumn{2}{|c|}{ SIMC } & \multicolumn{2}{c|}{ ITAE } & \multicolumn{2}{c}{ IMC-R } \\
\cline { 2 - 7 } & $K_{p}$ & $K_{i}$ & $K_{p}$ & $K_{i}$ & $K_{p}$ & $K_{i}$ \\
\hline \hline SIC-004 & 0,0739 & 0,0199 & 0,0463 & 0,0155 & 0,0203 & 0,0075 \\
FIC-013 & 0,4733 & 0,1760 & 0,2770 & 0,1337 & 0,1181 & 0,0660 \\
LIC-001 & 0,0531 & 0,0299 & 0,0231 & 0,0139 & 0,0092 & 0,0086 \\
SIC-001 & 0,0315 & 0,0091 & 0,0193 & 0,0071 & 0,0084 & 0,0034 \\
\hline \hline
\end{tabular}

\subsection{Metodologias Robustas}

Duas metodologias são empregadas neste trabalho para o cálculo dos controladores robustos que empregam um procedimento de modelagem para representar funções de transferências incertas com atraso para um sistema politópico em espaço de estados, da forma

$$
\dot{x}(t)=A(\alpha) x(t)+B(\alpha) u(t-\tau),
$$

tal que $\alpha$ é um parâmetro incerto pertencente ao simplex unitário (Koch et al., 2018) e os ganhos dos controladores PI são obtidos como um problema de realimentação de estados. A primeira, apresentada em Niculescu et al. (1997) e denominada como Robusto NF, garante a estabilidade para sistemas em malha fechada para atrasos de transporte suficientemente pequenos. A segunda, proposta em Oliveira (2018) e intitulada como Robusto GT, apresenta condições LMI baseadas em um funcional de LyapunovKrasovskii e no Lema de Finsler. A seguir, nos Teoremas 1 
e 2, são apresentadas as condições LMI utilizadas e as provas, omitidas por simplicidade, podem ser encontradas em Niculescu et al. (1997) e Oliveira (2018), respectivamente.

Teorema 1. Se existir uma matriz simétrica definida positiva $Q \in \mathbb{R}^{n \times n}$, uma matriz $W \in \mathbb{R}^{m \times n}$ e escalares $\beta_{1}$ e $\beta_{2}$ tal que

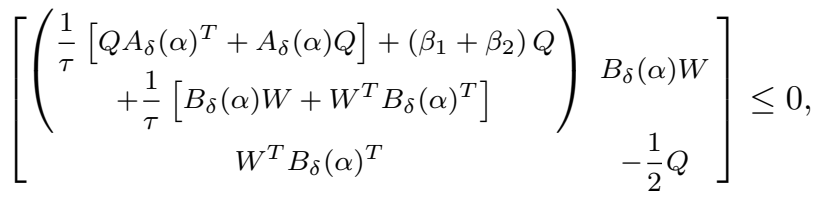

$$
\begin{aligned}
& {\left[\begin{array}{cc}
-\beta_{1} Q & A_{\delta}(\alpha) Q \\
Q A_{\delta}(\alpha)^{T} & -Q
\end{array}\right] \leq 0} \\
& \beta_{1}\left[\begin{array}{cc}
-Q & 0 \\
0 & 0
\end{array}\right]+\left[\begin{array}{cc}
0 & B_{\delta}(\alpha) W \\
W^{T} B_{\delta}(\alpha)^{T} & -Q
\end{array}\right] \leq 0
\end{aligned}
$$

sejam satisfeitas, com $A_{\delta}(\alpha)=A(\alpha)+\delta I$ e $B_{\delta}(\alpha)=$ $e^{\delta \tau(\alpha)} B(\alpha)$, então o sistema (2) em malha fechada é assintoticamente estável por meio de uma lei de controle do tipo $u(t)=K x(t), K \in \mathbb{R}^{m \times n}$, para todo atraso $\tau(t)$ que satisfaça $0 \leq \tau(t) \leq \tau$. Além disso, os ganhos do controlador são dados por $K=W Q^{-1}$.

Teorema 2. Se existirem matrizes simétricas definidas positivas $W \in \mathbb{R}^{n \times n}, X \in \mathbb{R}^{n \times n}$ e uma matriz $Z \in \mathbb{R}^{1 \times n} \mathrm{e}$ um dado escalar $\delta>0$ tal que

$$
\begin{gathered}
{\left[\begin{array}{ccc}
\Lambda_{11} & \tau \alpha^{2} B_{\delta}(\alpha) Z+\tau W & \tau^{2} W A_{\delta}(\alpha)^{T} \\
\star & -\tau^{2} X-\tau W & \tau^{2} Z B_{\delta}(\alpha)^{T} \\
\star & \star & -\tau W
\end{array}\right] \leq 0} \\
\operatorname{com} A_{\delta}(\alpha)=A(\alpha)+\delta I, B_{\delta}(\alpha)=e^{\delta \tau(\alpha)} B(\alpha) \mathrm{e} \\
\Lambda_{11}=\tau(\alpha)^{2}\left(W A_{\delta}(\alpha)^{T}+A_{\delta}(\alpha) W+X(\alpha)\right)-\tau(\alpha) W,
\end{gathered}
$$

seja satisfeita. Então o sistema (2) em malha fechada é assintoticamente estável com $K=Z W^{-1}$.

Para ambas as metodologias, utilizaram-se os parâmetros $K$ e $a$ apresentados em (1) e uma variação de $14 \%$ em relação aos modelos identificados e listados na Tabela 1. Definiu-se ainda uma região de alocação dos polos limitada por $\delta>0,11$, ou seja, todos os polos são alocados à esquerda de $-0,11$. Além disso, empregou-se a representação em espaço de estados e a descrição dos politopos conforme proposto em Koch et al. (2018), gerando politopos de quatro vértices.

Os ganhos $K_{p}$ e $K_{i}$ dos controladores obtidos para as malhas apresentadas na Tabela $1 \mathrm{com}$ as metodologias Robusto NF e robusto GT são os apresentados na Tabela 3.

Tabela 3. Ganho dos Controladores Robustos.

\begin{tabular}{c|cc|cc}
\hline \hline \multirow{2}{*}{ Malha } & \multicolumn{2}{|c|}{ Robusto NF } & \multicolumn{2}{c}{ Robusto GT } \\
\cline { 2 - 5 } & $K_{p}$ & $K_{i}$ & $K_{p}$ & $K_{i}$ \\
\hline \hline SIC-004 & 0,0365 & 0,0143 & 0,0437 & 0,0162 \\
FIC-013 & 0,1860 & 0,1150 & 0,2537 & 0,1301 \\
LIC-001 & 0,0189 & 0,0195 & 0,0295 & 0,0170 \\
SIC-001 & 0,0150 & 0,0065 & 0,0179 & 0,0071 \\
\hline \hline
\end{tabular}

\section{COMPARAÇÃO DAS METODOLOGIAS UTILIZADAS E RESULTADOS}

Nesta seção, apresenta-se uma comparação de cinco metodologias apresentadas para o cálculo dos ganhos $K_{p}$ e $K_{i}$ para o sistema de moagem descrito. A comparação entre os sinais foi realizada por meio de simulações de distúrbios e situações comuns que ocorrem na referida planta de moagem descrita por Reis (2018).

Descrito como experimento I, esse evento caracterizase pelo aumento do percentual de sólidos da polpa da alimentação da remoagem. O experimento II consiste em queda brusca da taxa de alimentação da usina. Finalmente, o experimento III descreve a variação da granulometria da alimentação da remoagem.

Tais eventos também foram modelados com o intuito de verificar a viabilidade de utilização de um controlador robusto nesse processo. Repetindo a mesma metodologia para a identificação da planta em condições sem distúrbio, verificou-se que o percentual de incertezas dos experimentos com distúrbios quando comparados com a planta sem o distúrbio, variavam de $3 \%$ para o experimento I, $14 \%$ para o experimento II e $18 \%$ para o experimento III.

Esses experimentos também foram observados por Galán et al. (2002) em seu estudo. Ele cita que condições de operação em um circuito de moagem são frequentemente tais que a produção é significativamente reduzida devido aos efeitos das perturbações do processo, principalmente associadas com o minério de alimentação.

O processo foi simulado utilizando o modelo validado por Reis (2018) no software IDEAS ${ }^{\mathrm{TM}}$, o controle do processo foi realizado no software MATLAB ${ }^{\circledR}$ e a comunicação entre eles foi realizada via OPC. O tempo de simulação foi de 3500s e igual para todos os experimentos, a amostragem dos dados foi de $0,1 \mathrm{~s}$.

Notou-se que para as malhas 02 e 03 não houve variação significativa no desvio dos valores medidos em relação as referências. As oscilações dos valores medidos foram inferiores a $0,01 \%$ nos três experimentos. Por isso não estão listados neste estudo os resultados dessas malhas.

\subsection{Experimento $I$}

Os resultados do experimento I aplicado à malha SIC004 e a malha SIC-001, estão mostrados nas Figuras 2 e 3, respectivamente. Para a malha SIC-004 (Figura 2), percebe-se que as metodologias robustas possuem tempo de acomodação aproximadamente iguais aos das metodologias clássicas não ultrapassando os limites inferior e superior de $2 \%$ do sinal de referência. Além disso, a metodologia Robusto NF possui um sobressinal $92 \%$ menor do que a metodologia clássica ITAE, os quais são os dois menores sobressinais para as sintonias robustas e clássicas, respectivamente. Percebe-se que, nesse caso, a metodologia Robusto NF obteve o menor sobressinal e a metodologia SIMC e ITAE foram as primeiras a alcançar a referência, apesar da metodologia SIMC se apresentar muito ruidosa. A metodologia Robusto GT alcança a referência no instante $300 \mathrm{~s}$ enquanto a metodologia SIMC, no tempo $170 \mathrm{~s}$.

Para a malha SIC-001 (Figura 3), percebe-se que as metodologias robustas e clássicas possuem o mesmo tempo de acomodação e não ultrapassam os limites inferior e superior de $2 \%$ do sinal de referência. Nota-se também que as metodologias Robustas alcançam a referência próximo ao tempo $900 \mathrm{~s}$ enquanto as metodologias clássicas alcançam 


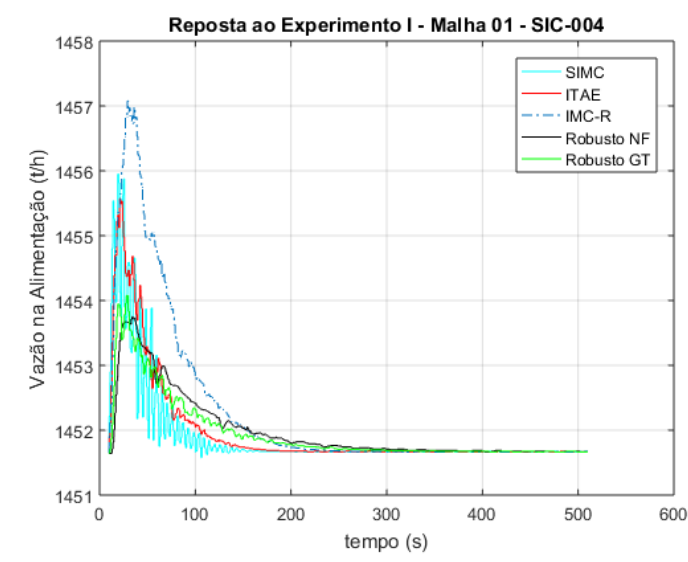

Figura 2. Resposta da malha SIC-004 ao experimento I para as metodologias utilizadas.

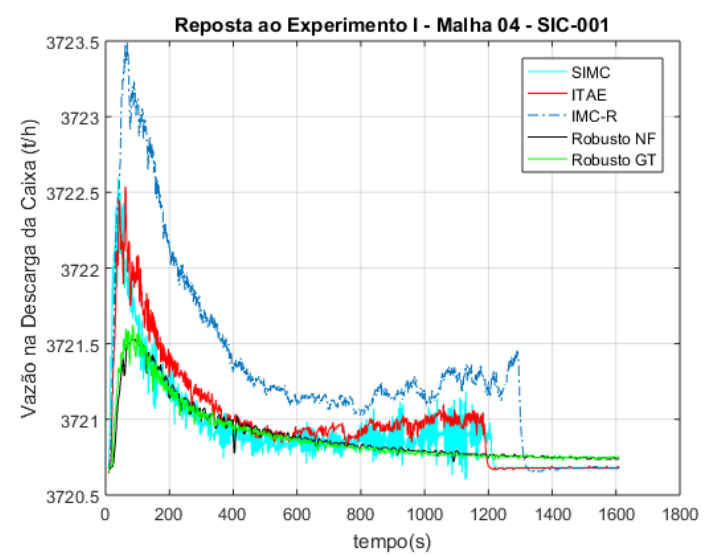

Figura 3. Resposta da malha SIC-001 ao experimento I para as metodologias utilizadas.

a partir de $1200 \mathrm{~s}$. Outra observação é que as metodologias robustas possuem um sobressinal $0,02 \%$ com relação a referência enquanto as clássicas tem um percentual de 0,05\% para o menor caso, ITAE. Percebe-se que nesse caso as metodologias Robusto NF e Robusto GT obtiveram resultados bem similares e que as metodologias ITAE e SIMC resultaram em sinais muito ruidosos se comparados aos demais.

\subsection{Experimento $I I$}

Os resultados do experimento II aplicados à malha SIC004 e a malha SIC-001, estão mostrados nas Figuras 4 e 5, respectivamente.

Para a malha SIC-004 (Figura 4), nota-se que as metodologias robustas e clássicas não ultrapassam os limites inferior e superior de $2 \%$ do sinal de referência. Outra observação importante refere-se ao sobressinal gerado pelos controladores robustos que é $0,58 \%$ enquanto para os clássicos $0,51 \%$ considerando o menor sobressinal dos dois tipos de metodologias. O controlador Robusto GT obteve o melhor resultado para essa malha. A metodologia clássica SIMC alcança a referência próximo de $150 \mathrm{~s}$ enquanto a metodologia Robusta GT alcança próximo ao tempo $275 \mathrm{~s}$. As metodologias ITAE e SIMC apresentam sinais ruidosos se comparadas as metodologias robustas.

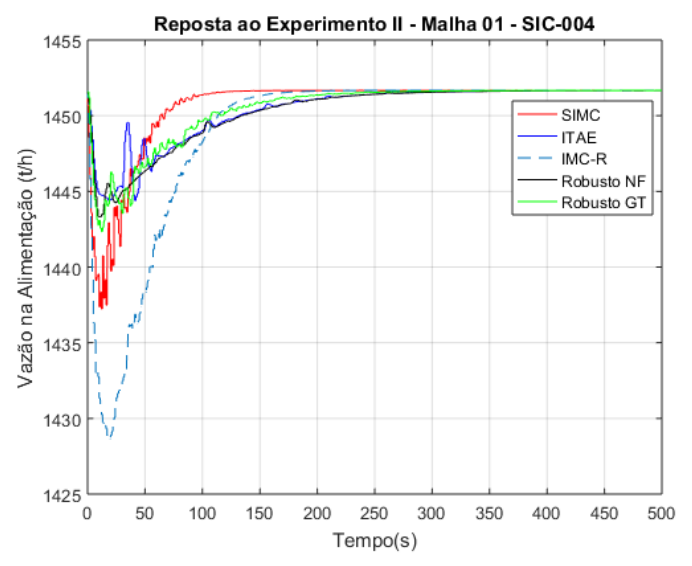

Figura 4. Resposta da malha SIC-004 ao experimento II para as metodologias utilizadas.

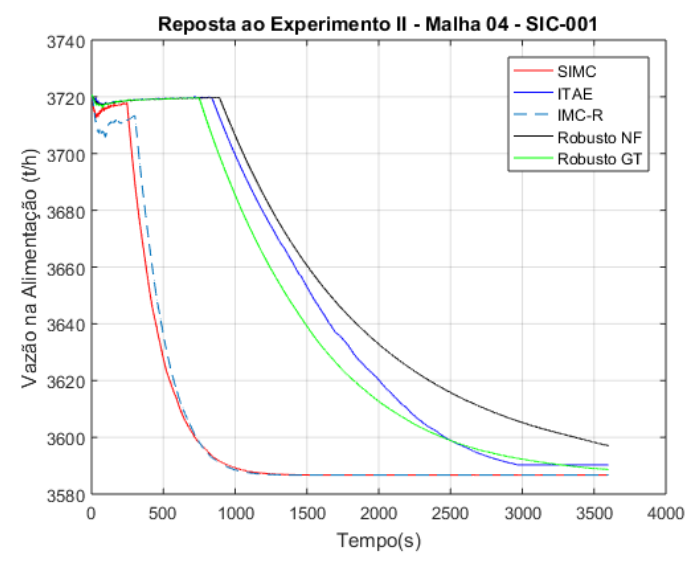

Figura 5. Resposta da malha SIC-001 ao experimento II para as metodologias utilizadas.

Para a malha SIC-001 (Figura 5), percebe-se que devido a outras condições do processo todas os controladores não são capazes de estabilizar o processo. Entretanto a metodologia Robusta NF é que consegue manter por maior tempo a estabilidade da malha e atinge os valores mais próximos a referência para esse processo perdurando pelo período de 0 a $900 s$.

\subsection{Experimento III}

$\mathrm{O}$ resultado do experimento III aplicado à malha SIC001 é mostrado na Figura 6. O resultado da malha SIC004 não está apresentado neste estudo pois as varições que ocorreram não foram significativas sendo inferiores a $0,01 \%$ do valor de referência e não justificando a análise desta malha. A análise do sobressinal indica que para as metodologias robustas foi de $0,01 \%$ da referência e 0,04\% para a metodologia SIMC, em ambos considerouse o menor sobressinal das metodologias testadas. As metodologias clássicas para esta malha tornam-se instáveis a partir do tempo $1400 \mathrm{~s}$ enquanto as robustas mantêm a estabilidade até aproximadamente o tempo de $3500 \mathrm{~s}$.

Para esse experimento especificamente a função de transferência que o modelou tinha variações de $17 \%$ se comparada ao modelo da planta. Pode-se inferir que neste caso, mesmo que a incerteza do sistema seja maior que a projetada é possível que o controlador robusto estabilize o sistema. 


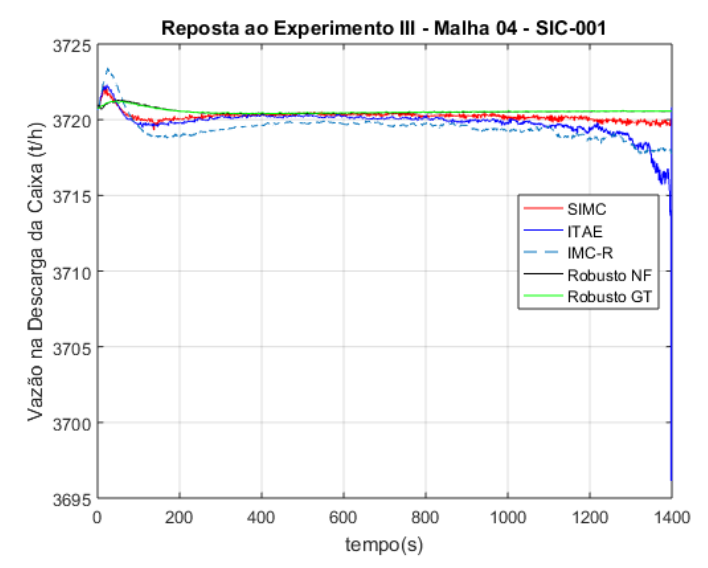

Figura 6. Resposta da malha SIC-001 ao experimento III para as metodologias utilizadas.

\section{CONCLUSÃO E TRABALHOS FUTUROS}

Neste trabalho foi estudado o projeto de controladores PI clássicos e robustos para o processo na moagem. Apresentaram-se os principais equipamentos da moagem, as variações e incertezas que fazem parte do processo e dos equipamentos. Tais variações influenciam o processo e o uso de controladores robustos apresenta-se como uma estratégia viável prática. Foram propostos três experimentos que ocorrem na planta da Samarco e analisados os comportamentos do processo ante aos controladores. Notou-se que, de modo geral, os controladores robustos possuem menor sobressinal e, se comparados com os clássicos, possuem sinais menos ruidosos. Foi possível com os controladores robustos estabilizar o sistema cujos modelos possuíam maior incerteza politópica do que a projetada enquanto que para as metodologias clássicas não foi possível. Como trabalho futuro pretende-se comparar os resultados obtidos como outros modelos e considerar um estratégia de controle em cascata para o mesmo processo.

\section{REFERÊNCIAS}

Castro, F.F. e Braga, M.F. (2018). Estudo comparativo entre controladores PID e MPC aplicados a moinhos de bolas para beneficiamento de minério. In $A B M W e e k, \mathrm{p}$. 435-443. São Paulo.

Chen, X.S., Li, S.H., Zhai, J.Y., e Li, Q. (2009). Expert system based adaptive dynamic matrix control for ball mill grinding circuit. Expert Systems with Applications, $36(1), 716-723$.

Chien, K.L., Hrones, J.A., e Reswick, J.B. (1952). On the automatic control of generalized passive systems. Trans. ASME, 74, 175-185.

Galán, O., Barton, G., e Romagnoli, J. (2002). Robust control of a SAG mill. Powder Technology, 124(3), 264 - 271. Control of Particulate Processess IV.

Guerrero, F., Bouchard, J., Poulin, E., e Sbarbaro, D. (2016). Real-time simulation and control of a SAG mill. IFAC-PapersOnLine, 49(20), 61 - 66. 17th IFAC Symposium on Control, Optimization and Automation in Mining, Mineral and Metal Processing MMM 2016.

Hongwei, Z., Yiming, Q., Jiye, Z., e Yuanheng, Z. (2010). Based on multi-agent model for grinding process control research. Proc. 5th Int. Conf. Frontier of Comput. Sci. Technol. (FCST), 576-581.
Jenkins, H.E., Kurfess, T.R., e Richard C. Dorf, F. (1996). Design of a robust controller for a grinding system. IEEE Transactions On Control Systems Technology, 4(1), 4049.

Koch, G.G., Gabbi, T.S., Vieira, R.P., Pinheiro, H., Bernardes, T.A., Oliveira, R.C.L.F., e Montagner, V.F. (2018). Linear matrix inequality based synthesis of PI controllers for PMSM with uncertain parameters. Revista Eletrônica de Potência, Volume 23(3), 310-319.

le Roux, J.D., Padhi, R., e Craig, I.K. (2014). Optimal control of grinding mill circuit using model predictive static programming: A new nonlinear MPC paradigm. Journal of Process Control, 24(12), 29 - 40.

Légaré, B., Bouchard, J., e Poulin, E. (2016). A modular dynamic simulation model for comminution circuits. IFAC Conference Paper Archive.

Lopes Jr., Ê., Ferreira, A., Moreira, V., e Euzébio, T. (2018). Fuzzy fault tolerant controller applied in an iron ore concentrate dewatering plant. In $6^{\text {th }}$ International Congress on Automation in Mining. GECAMIN.

Magalhães, S. e Euzébio, T. (2018). Supervisory fuzzy controller for thickener underflow solids concentration on a simulated platform. In $6^{\text {th }}$ International Congress on Automation in Mining. GECAMIN.

Niculescu, S.i., Fu, M., e Li, H. (1997). Delay dependent closed loop stability of linear systems with input delay: An LMI approach. IEEE Conference on Decision and Control.

Oliveira, G.A. (2018). Projeto de Controladores PID Robustos para Sistemas Politópicos Sujeitos à Atraso Incerto: Uma Abordagem LMI. Dissertação de Mestrado, UnB, Brasília, DF.

Reis, L., Júnior, Ê.L., e Euzébio, T. (2018). Hardwarein-the-loop testing platform of a predictive controller applied to a simulated regrinding circuit. In $6^{\text {th }}$ International Congress on Automation in Mining. GECAMIN.

Reis, L.A. (2018). Controle de um circuito simulado de remoagem de minério de ferro por controle preditivo por modelo. Dissertação de Mestrado, Universidade Federal de Ouro Preto and Instituto Tecnológico Vale, Ouro Preto.

Rockwell Automation, I. (2016). Otimização utilizando controle preditivo multivariável em mineração. Technical report. Publicação MIN-BR001A-PT-E Janeiro 2016.

Sbárbaro, D. e del Villar, R. (eds.) (2010). Advanced Control and Supervision of Mineral Processing Plants. Springer.

Seborg, D.E., Mellichamp, D.A., Edgar, T.F., e Doyle, F.J. (2010). Process Dynamics and Control. John Wiley \& Sons.

Silva, J.P. (2014). Caracterização dos Hidrociclones Utilizados nas Etapas de Classificação dos Concentradores I e II da Samarco Mineração. Dissertação de Mestrado, Universidade Federal de Ouro Preto.

Skogestad, S. (2003). Simple analytic rules for model reduction and pid controller tuning. J. Process Control, 13, 291-309.

Su, J., Zhang, X., Zeng, X., e Cui, Y. (2009). Design and simulation of robust ball grinding mill control system. In 2009 Chinese Control and Decision Conference, 28132818. 